\title{
质子惰性介质中芳香族硝基化合物在铂微电极上的电化学行为
}

\author{
屠晓华 ${ }^{1,2,3}$ 褚有群 ${ }^{2}$ 马淳安 ${ }^{2, *}$ \\ ('嘉兴学院生物与化学工程学院, 浙江 嘉兴 314001; 2 2 浙江工业大学应用化学系, 绿色化学合成技术国家重点实验室 \\ 培育基地, 杭州 310032； 3 嘉兴市化工清洁工艺重点实验室, 浙江 嘉兴 314001)
}

\begin{abstract}
摘要: 采用循环伏安法 $(C V)$ 和电化学阻抗谱 $(E I S)$ 技术, 以铂微电极为工作电极, 大面积铂丝为对电极, 饱和 甘录电极为参比电极, 研究了不同芳香族硝基化合物(NA)(硝基苯、硝基萗和间二硝基苯)在含有四丁基高氯酸 铵(TBAP) 电解质的 $N, N$-二甲基甲酰胺(DMF)有机溶液中的电化学行为, 并探讨了扫描速率、硝基数目和苯环 数目等因素对硝基电化学还原特性的影响. 结果表明: 三种芳香族硝基化合物在铂微电极上的反应均为扩散控 制的准可逆过程; 苯环上硝基数目的增加容易使硝基在较低电位下还原; 苯环数目的增加导致硝基的还原峰电 流急剧减小.
\end{abstract}

关键词：芳香族硝基化合物; 微电极; 质子惰性介质; 循环伏安; 电化学阻抗谱 中图分类号: 0646

\section{Electrochemical Behavior of Nitroaromatics on Pt Microelectrode in an Aprotic Medium}

\author{
TU Xiao-Hua ${ }^{1,2,3}$ CHU You-Qun ${ }^{2} \quad$ MA Chun-An ${ }^{2, *}$ \\ ( ${ }^{1}$ College of Biological and Chemical Engineering, Jiaxing University, Jiaxing 314001, Zhejiang Province, P. R. China; \\ ${ }^{2}$ State Key Laboratory Breeding Base of Green Chemistry-Synthesis Technology, Department of Applied Chemistry, Zhejiang \\ University of Technology, Hangzhou, 310032, P. R. China; ${ }^{3}$ Key Laboratory of Clean Chemical Process of Jiaxing, \\ Jiaxing 314001, Zhejiang Province, P. R. China)
}

\begin{abstract}
N}, \mathrm{N}$-dimethylformamide (DMF) and tetrabutyl ammonium perchlorate (TBAP) were used as a solvent and a supporting electrolyte, respectively. The working electrode was a platinum microelectrode, the auxiliary electrode was a large area platinum wire and the reference electrode was a saturated calomel electrode. The electrochemical behavior of nitroaromatics (NA) was studied by cyclic voltammetry (CV) and electrochemical impedance spectroscopy (EIS). The effects of scan rate, numbers of benzene rings and nitro groups on the electrochemical reduction of NA were investigated systematically. The results showed that the reaction of NA on the Pt microelectrode was a quasi-reversible process controlled by the diffusion of NA. We found that NA was more electrochemically reducible when it contained more nitro groups and the reduction peak current of the nitro group decreased in the presence of more benzene rings.
\end{abstract}

Key Words: Nitroaromatics; Microelectrode; Aprotic medium; Cyclic voltammetry; Electrochemical impedance spectroscopy

1 引 言

芳香族硝基化合物的电化学还原是一个十分
重要的有机电化学反应. 早在二十世纪初, Haber 在 研究硝基苯的分步还原时首次提出电极电位才是

Received: March 22, 2011; Revised: May 27, 2011; Published on Web: July 7, 2011.

"Corresponding author. Email: science@zjut.edu.cn; Tel: +86-571-88320830.

The project was supported by the Special Prophase Project on Basic Research of National Department of Science and Technology (2003CCA01300) and National Natural Science Foundation of China (20376074).

国家基础研究重大项目前期研究专项(2003CCA01300)和国家自然科学基金(20376074)资助项目

(C) Editorial office of Acta Physico-Chimica Sinica 
决定电解产物的主要因素, 从而为有机电化学合成 研究指明了方向, 极大地推动了有机电化学工业的 发展. 由于在不同条件下电化学还原芳香族硝基化 合物可制得相应的胺、偶氮化合物、肼、羟胺等一系 列具有重要应用价值的有机化合物, 迄今为止, 该 类化合物的电化学还原仍受到人们的广泛关注. 但 对芳香族硝基化合物的研究大多限于单环单硝基 化合物电化学还原特性研究, 对多硝基取代苯及多 环硝基化合物的研究较少有文献报道. ${ }^{1-11}$ 这类研究 大多涉及质子介质体系, 而硝基的还原是一类需要 质子供应的反应, 因此, 由于大量质子的存在, 质子 溶剂中芳香族硝基化合物的电化学还原过程很难 捕捉到硝基还原中间态的过程, 而质子惰性介质由 于很好地隔离了质子的影响, 对于研究复杂的电化 学中间步骤显得非常方便. ${ }^{12-15}$

微电极技术在电化学科学实验中已经被广泛 地运用起来, 相对于一般的常规电极, 微电极具有 多方面的特点. ${ }^{16}$ 因此, 微电极对于研究非水体系的 电化学行为极为适用..$^{7-21}$ 本文采用铂微电极为工作 电极, 结合循环伏安法和电化学阻抗谱技术, 在质 子惰性介质 $N, N$-二甲基甲酰胺(DMF)中, 以四丁基 高氯酸铵(TBAP) 为支持电解质, 研究了不同苯环 数、不同硝基数对芳香族硝基化合物电化学还原特 性的影响.

\section{2 实验部分}

\section{1 铂微电极的制备}

将直径 $60 \mu \mathrm{m}$ 的铂丝一端与铜线相连, 另一端 与玻璃管烧结封口, 将封有铂丝的一端用 $\mathrm{Al}_{2} \mathrm{O}_{3}$ 粉抛 光磨成镜面, 然后置于二次蒸馏水中超声波清洗, 即制成铂微电极.

\section{2 电化学测试}

电化学测试所用的仪器为 AutoLab PGSTAT 30
型电化学工作站(荷兰), 测试工作在三电极电解池 中进行. 工作电极为铂微电极, 对电极为大面积铂 丝, 参比电极为饱和甘录电极(SCE), 在本文中提到 的电位都相对于 SCE.

电解液为含有不同芳香族硝基化合物的四丁 基高氯酸铵 $\left(0.10 \mathrm{~mol} \cdot \mathrm{L}^{-1}\right)-N, N$-二甲基甲酰胺 (DMF)溶液. 所用试剂均为分析纯试剂(国药集团化 学试剂有限公司). 实验测试在室温下进行, 测试前 电解液先通高纯氮气 $30 \mathrm{~min}$, 以除去电解液中的溶 解氧.

\section{3 结果与讨论}

\section{1 芳香族硝基化合物在 DMF 中的循环伏安行为}

在微电极上通过扫描得到的伏安曲线依赖于 扫描速率, 在较低的扫描速率下, 一般呈 “S” 状. 这 是由于电活性物质到微电极表面的传质速率非常 高, 因而在微电极/溶液界面附近的浓度在缓慢的电 位扫描过程中瞬间即可达到稳态. 从而需要达到一 定的扫描速率才能在伏安曲线上出现氧化还原峰, 因此在进行循环伏安扫描时, 选取扫描速率为 100 $\mathrm{mV} \cdot \mathrm{s}^{-1}$. 图 $1 \mathrm{a}$ 为质子惰性介质中硝基苯在铂微电极 上的循环伏安曲线, 其中曲线 1 是在室温下含 0.1 $\mathrm{mol} \cdot \mathrm{L}^{-1} \mathrm{TBAP}$ 的 $\mathrm{DMF}$ 溶液的 $\mathrm{CV}$ 曲线, 曲线 2 为在 $\mathrm{TBAP} / \mathrm{DMF}$ 体系中加入硝基苯后的 $\mathrm{CV}$ 曲线, 在 $0.20--2.00 \mathrm{~V}$ 扫描电位区间出现了一对氧化还原 峰, 氧化峰和还原峰形状基本对称. 根据前人的研 究结果, ${ }^{12-15}$ 可以认为硝基苯在 DMF 溶液中的电极 反应为单电子转移的准可逆过程, 对应于芳香族硝 基化合物及其阴离子自由基的氧化还原过程, 如(1) 式所示:

$$
\mathrm{ArNO}_{2}+\mathrm{e} \rightleftharpoons \mathrm{ArNO}_{2}^{-} \cdot
$$

图 $1 \mathrm{~b}$ 为质子惰性介质中硝基荟在铂微电极上 的循环伏安曲线, 从图中可以得到硝基萗在质子惰
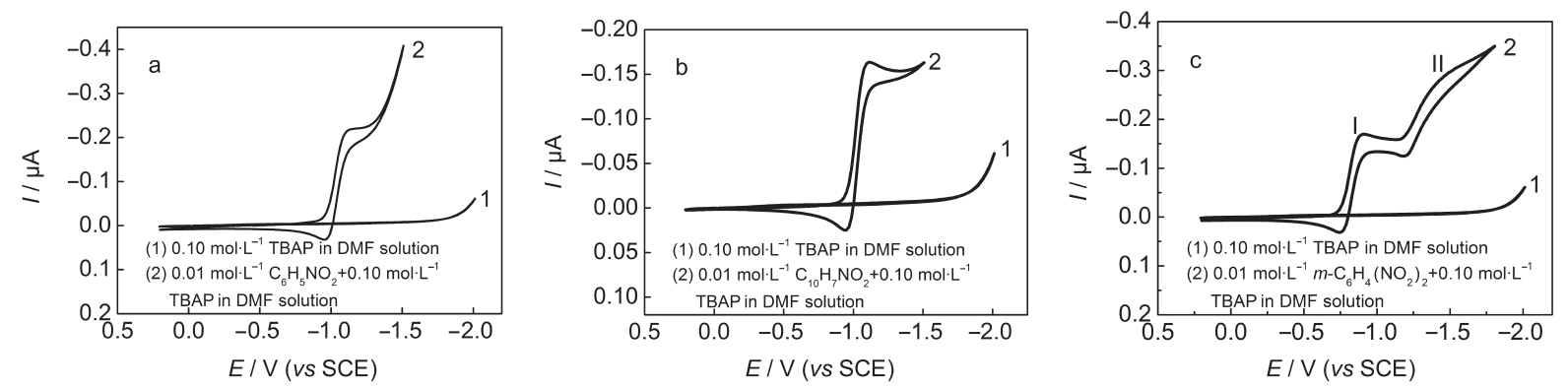

图 1 芳香族硝基化合物(NA)在铂微电极上的循环伏安曲线

Fig.1 CV curves of nitroaromatics (NA) on Pt microelectrode

TBAP: tetrabutyl ammonium perchlorate, DMF: $N, N$-dimethylformamide, $\nu=100 \mathrm{mV} \cdot \mathrm{s}^{-1}$ 

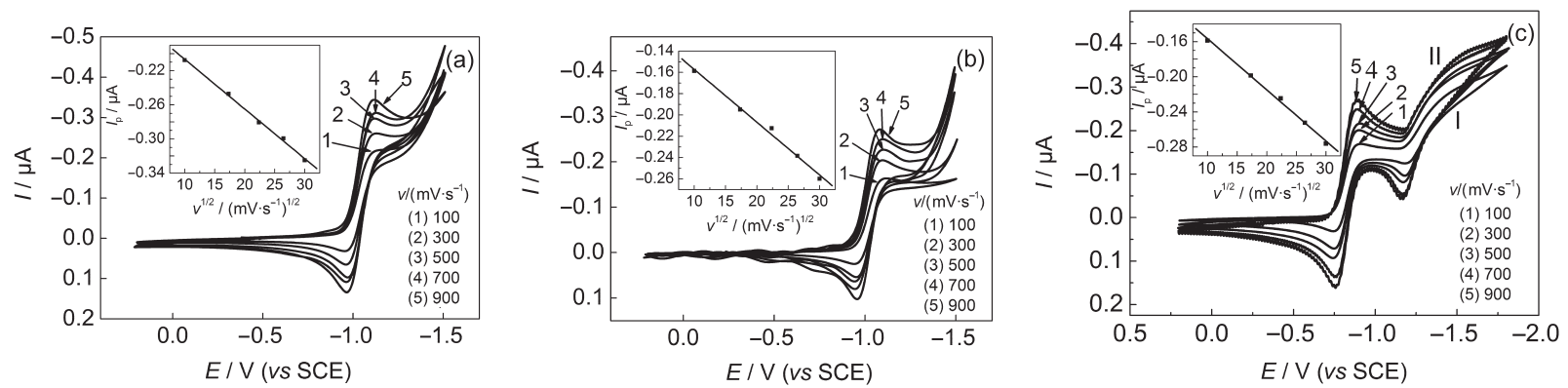

图 2 不同芳香族硝基化合物的 DMF 溶液在铂微电极上不同扫描速率时的循环伏安曲线

Fig.2 CV curves of Pt microelectrode in NA-TBAP-DMF at various scan rates

$c($ TBAP $)=0.10 \mathrm{~mol} \cdot \mathrm{L}^{-1}, c(\mathrm{NA})=0.01 \mathrm{~mol} \cdot \mathrm{L}^{-1}$; (a) $\mathrm{C}_{6} \mathrm{H}_{5} \mathrm{NO}_{2}$, (b) $\mathrm{C}_{10} \mathrm{H}_{7} \mathrm{NO}_{2}$, (c) $m-\mathrm{C}_{6} \mathrm{H}_{4}\left(\mathrm{NO}_{2}\right)_{2}$

性介质中的氧化还原过程也同样属于准可逆过程. 因此硝基萗的氧化还原过程同硝基苯一样, 可能对 应于芳香族硝基化合物和芳香族硝基化合物阴离子 自由基的氧化还原过程, 如(1)式所示. 图 1c 是质子 惰性介质中间二硝基苯在铂微电极上的循环伏安 图, 在 $0.20--2.00 \mathrm{~V}$ 扫描电位区间出现了两对氧化 还原峰, 这可能可以归结为间二硝基苯 $\left(\mathrm{O}_{2} \mathrm{NPhNO}_{2}\right)$ 的分步还原, 峰 I 对应于 $\mathrm{O}_{2} \mathrm{NPhNO}_{2}$ 以单电子反应还 原为 $\mathrm{O}_{2} \mathrm{NPhNO}_{2}^{-}$的过程, 峰 II 对应于 $\mathrm{O}_{2} \mathrm{NPhNO}_{2}^{-}$通 过单电子反应还原成 $\left[\mathrm{O}_{2} \mathrm{NPhNO}_{2}\right]^{2-}$ 的过程, 同时伴 随有 $\mathrm{O}_{2} \mathrm{NPhNO}_{2}$ 在铂微电极上直接或分步连续的通 过总电子数为 2 的反应还原为 $\left[\mathrm{O}_{2} \mathrm{NPhNO}_{2}\right]^{2-}$ 的过程, 峰 II 是该两种电极过程的叠加, 由于在电极表面 $\mathrm{O}_{2} \mathrm{NPhNO}_{2}$ 的浓度小于 $\mathrm{O}_{2} \mathrm{NPhNO}_{2}^{-}$的浓度, 因此 $\mathrm{O}_{2} \mathrm{NPhNO}_{2}^{-}$得到一个电子还原为 $\left[\mathrm{O}_{2} \mathrm{NPhNO}_{2}\right]^{2-}$ 的过 程是峰 II 的主要电极过程. 该氧化还原过程可以用 下述过程表示:

$$
\begin{aligned}
& \mathrm{O}_{2} \mathrm{NPhNO}_{2}+\mathrm{e} \rightleftharpoons \mathrm{O}_{2} \mathrm{NPhNO}_{2}^{-} \\
& \mathrm{O}_{2} \mathrm{NPhNO}_{2}^{-}+\mathrm{e} \rightleftharpoons\left[\mathrm{O}_{2} \mathrm{NPhNO}_{2}\right]^{2-} \\
& \mathrm{O}_{2} \mathrm{NPhNO}_{2}+2 \mathrm{e} \rightleftharpoons\left[\mathrm{O}_{2} \mathrm{NPhNO}_{2}\right]^{2-}
\end{aligned}
$$

\section{2 扫描速率对循环伏安曲线的影响}

图 2 为不同芳香族硝基化合物在不同扫描速率 下的 CV 曲线, 随着扫描速率的增加, 峰电流增大, 还原峰电位负移, 氧化峰电位正移, 但氧化峰和还 原峰仍旧都保持了较好的可逆性. 其中, 对应于硝 基苯还原峰电流 $\left(I_{\mathrm{p}}\right)$ 与扫描速率 $v^{1 / 2}$ 拟合的直线方程 为

$$
I_{\mathrm{p}}=-5.83 v^{1 / 2} \times 10^{-9}-1.48 \times 10^{-7} \quad(R=0.9986)
$$

硝基萗还原峰电流 $\left(I_{\mathrm{p}}\right)$ 与扫描速率 $v^{1 / 2}$ 拟合的直线方 程为

$$
I_{\mathrm{p}}=-4.95 v^{1 / 2} \times 10^{-9}-1.08 \times 10^{-7} \quad(R=0.9957)
$$

速率 $v^{1 / 2}$ 拟合的直线方程为

$$
I_{\mathrm{p}}=-5.82 v^{1 / 2} \times 10^{-9}-9.86 \times 10^{-8} \quad(R=0.9982)
$$

由以上直线方程可知, $I_{\mathrm{p}}$ 与 $v^{1 / 2}$ 成正比, 说明芳香族硝 基化合物在电极界面的扩散为线性扩散, 电极过程 受芳香族硝基化合物向电极/溶液界面的扩散所控 制.

\section{3 不同硝基数对循环伏安曲线的影响}

图 3 为硝基苯和间二硝基苯在铂微电极上的循 环伏安曲线. 由图 3 可知, 间二硝基苯还原峰电位 (峰 I) 相对于硝基苯的还原峰电位正移了大约 0.23 $\mathrm{V}$, 峰 II 则负移 $0.20 \mathrm{~V}$ 左右. 间二硝基苯两个硝基的 存在直接导致了还原峰电位的偏移. 硝基是一个强 吸电子基团, 它的强吸电子作用直接导致了另一个 硝基上面电子云密度减小, 从而正电性增加. 因此, 相对于硝基苯, 间二硝基苯只需在更低电位下就能 获得一个电子生成带一个负电荷的硝基阴离子自 由基. Charoenkwan 等 ${ }^{12}$ 认为, 不带电荷的中性分子 相比已经带一个负电荷的阴离子自由基, 更容易获 得电子被还原. 由式(3)可知, 间二硝基苯还原峰 II

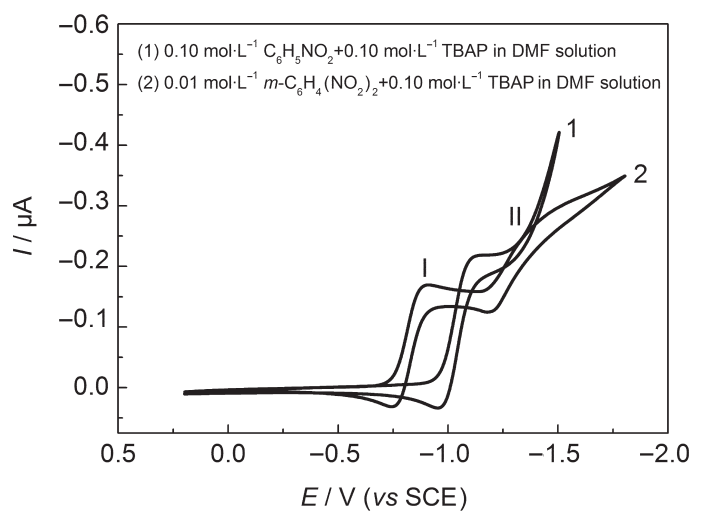

图 3 硝基苯和间二硝基苯在铂微电极上的循环伏安曲线

Fig.3 CV curves of $\mathrm{Pt}$ microelectrode in $\mathrm{C}_{6} \mathrm{H}_{5} \mathrm{NO}_{2}$ TBAP-DMF and $m-\mathrm{C}_{6} \mathrm{H}_{4}\left(\mathrm{NO}_{2}\right)_{2}$-TBAP-DMF solutions $v=100 \mathrm{mV} \cdot \mathrm{s}^{-1}$ 


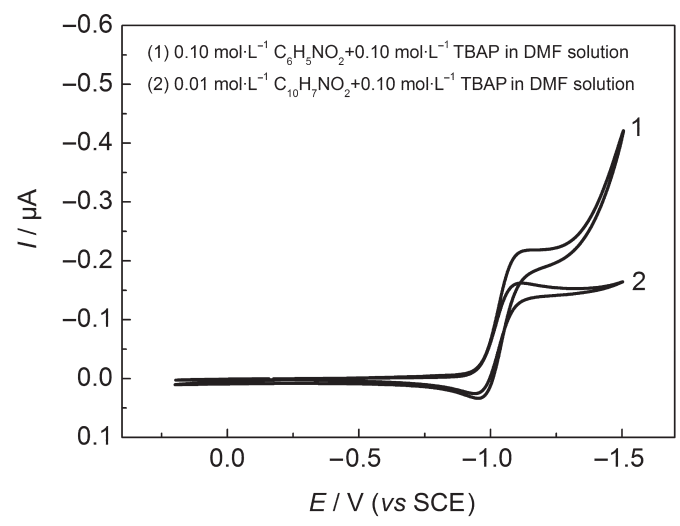

图 4 硝基苯和硝基萗在铂微电极上的循环伏安曲线 Fig.4 CV curves of $\mathrm{Pt}$ microelectrode in $\mathrm{C}_{6} \mathrm{H}_{5} \mathrm{NO}_{2}$ TBAP-DMF and $\mathrm{C}_{10} \mathrm{H}_{7} \mathrm{NO}_{2}$-TBAP-DMF solution $v=100 \mathrm{mV} \cdot \mathrm{s}^{-1}$

的电极过程对应于 $\mathrm{O}_{2} \mathrm{NPhNO}_{2}^{-*}$ 得一个电子还原为 $\left[\mathrm{O}_{2} \mathrm{NPhNO}_{2}\right]^{2-}$, 而硝基苯可以认为是不带电荷的中 性分子, 因此在同样条件下, 硝基苯比带一个负电 荷的间二硝基苯阴离子自由基更容易获得电子被 还原. 因此从 CV 图上反应出来间二硝基苯第二个 硝基的还原需要在更负的电位下才能进行, 也就是 需要在施加更强的外界条件才能发生硝基阴离子 的还原反应.

\section{4 不同苯环数对循环伏安曲线的影响}

图 4 为硝基苯和硝基荎在铂微电极上的循环伏 安曲线. 由图 4 可以得到, 硝基苯还原峰峰电流比硝 基荟大. 硝基萗还原峰电流的减小, 主要是由于苯 环数目的增加, 导致了硝基菜在溶液中的液相传质 阻力大于硝基苯, 以及在电极/溶液界面的扩散阻力 明显大于硝基苯. 由于硝基萗在溶液中的传质没有

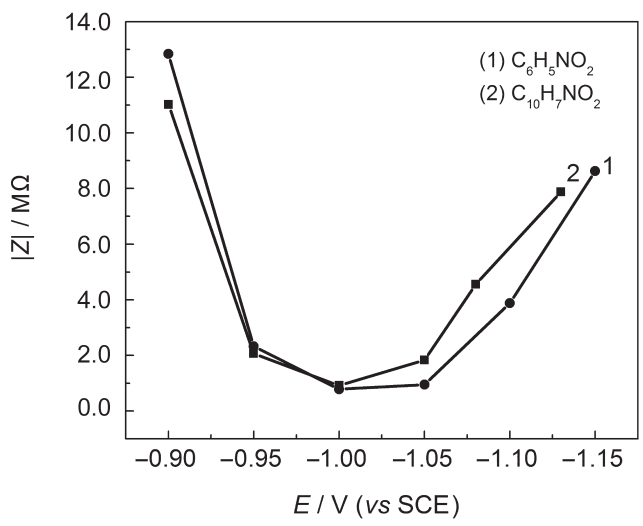

图 5 不同电位下芳香族硝基化合物的阻抗曲线

Fig.5 Plots of impedance of nitroaromatics at different voltages

$0.01 \mathrm{~mol} \cdot \mathrm{L}^{-1} \mathrm{NA}+0.10 \mathrm{~mol} \cdot \mathrm{L}^{-1} \mathrm{TBAP}$ in DMF solution at $10 \mathrm{mHz}$ on Pt microelectrode

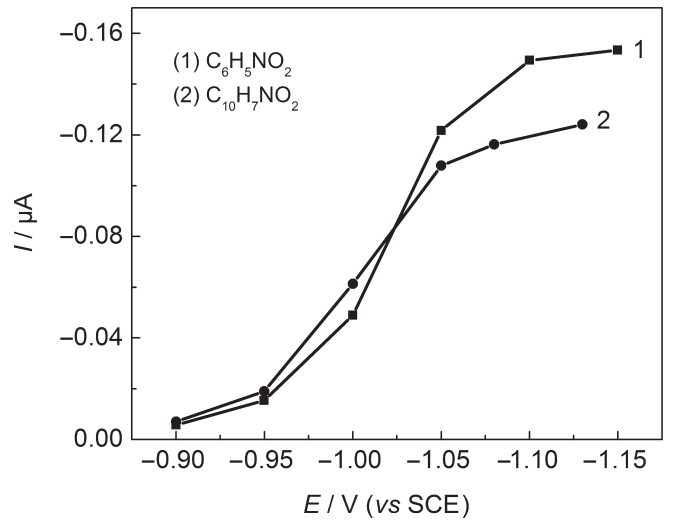

图 6 不同电位下芳香族硝基化合物的伏安曲线

Fig.6 Voltammograms of nitroaromatics at different voltages

$0.01 \mathrm{~mol} \cdot \mathrm{L}^{-1} \mathrm{NA}+0.10 \mathrm{~mol} \cdot \mathrm{L}^{-1} \mathrm{TBAP}$ in DMF solution at $10 \mathrm{mHz}$ on Pt microelectrode

硝基苯快, 因此在同样条件下, 随着硝基还原反应 的进行, 在电极表面硝基苯的浓度要高于硝基荎的 浓度; 并且, 随着苯环数的增加导致芳环上共轭效 应增强, 从而致使硝基萗上硝基的正电性降低. 所 以从 $\mathrm{CV}$ 曲线上反应出来硝基苯的峰电流要高于硝 基荟的峰电流. 图 5 是频率为 $10 \mathrm{mHz}$ 时不同电位下 的阻抗图, 由图 5 可以得到在 $-1.00--1.15 \mathrm{~V}$ 电位 区间硝基萗的阻抗值明显大于硝基苯, 从而说明硝 基萗从本体溶液扩散到电极表面更加困难, 这也对 应了图 4 中在 $-1.00--1.15 \mathrm{~V}$ 电位区间上硝基苯的 峰电流要高于硝基萘. 图 6 为 $10 \mathrm{mHz}$ 时不同电位下 的伏安曲线, 从图 6 中可知, 在 $-1.00--1.15 \mathrm{~V}$ 电位 区间上的硝基苯的还原电流明显大于硝基菱的还 原电流, 这与图 4 获得的结果一致.

\section{4 结 论}

研究了在含有四丁基高氯酸铵电解质的 $N, N$ 二甲基甲酰胺溶液中, 芳香族硝基化合物在铂微电 极上的电化学行为. 结果表明: 在所研究的条件下, 芳香族硝基化合物的还原过程属于动力学的准可 逆过程, 电极反应的控制步骤为扩散控制步骤; 由 于硝基是个强吸电子基团, 所以随着硝基数的增 加, 间二硝基苯第一个硝基, 相比硝基苯, 只需在更 低电位下就能获得电子生成硝基阴离子自由基; 随 着苯环数目的增加, 硝基还原峰电流急剧减小, 主 要是由于苯环数目的增加, 导致芳香族硝基化合物 在溶液/电极表面的扩散阻力明显增大, 以及共轭效 应增强降低了芳环上硝基的正电性. 


\section{References}

(1) Jiang, J. H.; Chen, L.; Wu, B. L.; Zhai, R. S. Acta Phys. -Chim. Sin. 1998, 14 (8), 704 . [江军华, 陈 岗, 吴秉亮, 翟润生. 物理化学学报, 1998, 14 (8), 704.]

(2) Ma, C. A.; Ge, X. F.; Zhu, Y. H.; Wang, L. B. J. Chem. Eng. Chin. Univ. 2006, 20, 728 . [马淳安, 葛小芳, 朱英红, 王连邦. 高校化学工程学报, 2006, 20,728.]

(3) Ma, C. A.; Tang, J. Y.; Li, G. H.; Sheng, J. F. Acta Chin. Sin. 2006, 64 (20), 2123. [马淳安, 汤俊艳, 李国华, 盛江峰. 化学学报, 2006, 64 (20), 2123.]

(4) Ma, C. A.; Huang, Y.; Tong, S. P.; Zhang, W. M. Acta Phys. -Chim. Sin. 2005, 21 (7), 721. [马淳安, 黄 烨, 童少 平, 张维民. 物理化学学报, 2005, 21 (7), 721 .]

(5) Ma, C. A.; Huang, X. Y.; Chu, Y. Q.; Tu, X. H.; Wang, S. Q. J. Zhejiang Univ. Tech. 2007, 35, 591. [马淳安, 黄祥岳, 褚有 群, 屠晓华, 王素琴. 浙江工业大学学报, 2007, 35, 591.]

(6) Ma, C. A.; Chu, Y. Q.; Zhu, Y. H.; Xu, Z. H. Electrochemistry 2004, 10 (3), 298. [马淳安, 褚有群, 朱英红, 徐志花. 电化学, 2004, 10 (3), 298.]

(7) Teng, W. J.; Mao, X. B.; Ma, C. A. J. Chem. Ind. Eng. 2010, 61 (5), 1313. [滕文娟, 毛信表, 马淳安. 化工学报, 2010, 61 (5), 1313.]

(8) Ma, C. A.; Chu, Y. Q.; Tong, S. P.; Mao, X. B.; Li, M. C.; Zhu, Y. H. J. J. Chem. Ind. Eng. 2004, 55 (12), 1971. [马淳安, 褚有 群, 童少平, 毛信表, 李美超, 朱英红. 化工学报, 2004, 55 (12), 1971.]

(9) Li, M. C.; Wu, H. F.; Hu, J. Q.; Ma, C. A. Acta Phys. -Chim. Sin. 2008, 24 (10), 1937. [李美超, 吴海峰, 胡佳琦, 马淳安. 物理
化学学报, 2008, 24 (10), 1937.]

(10) Zhang, X.; Liu, Z.; Dong, Y. M.; Zhou, C. L. J. Univ. Jinan (Sci \& Tec.), 2009, 23 (4), 398. [张 釒金, 刘 震, 董䎦敏, 周长利. 济南大学学报(自然科学版), 2009, 23 (4), 398.]

(11) Ma, Y. J.; Fu, Z. Z.; Ren, X. N.; Song, Q. Y.; Zhou, X. Y. Chin. J. Anal. Chem. 2008, 36 (2), 241. [马永均, 付周周, 任小娜, 宋青云, 周秀英. 分析化学, 2008, 36 (2), 241.]

(12) Charoenkwan, K.; Dennis, H. E. J. Electroanal. Chem. 2004, $565,29$.

(13) Núñez-Vergara, L. J.; Bonta, M.; Navarrete-Encina, P. A.; Squella, J. A. Electrochimica Acta 2001, 46, 4289.

(14) Bontá, M.; Chauviere, G.; Périé, J.; Núñez-Vergara, L. J.; Squella, J. A. Electrochimica Acta 2002, 47, 4045.

(15) Núñez-Vergara, L. J.; Ortiz, M. E.; Bollo, S.; Squella, J. A. Chem. Biol. Interact. 1997, 106, 1

(16) Goncalves, A.; Mathieu, C.; Herlem, M.; Etcheberry, A. J. Electroanal. Chem. 1999, 477, 140.

(17) Oldham, K. B.; Cardwell, T. J.; Santos, J. H.; Bond, A. M. J. Electroanal. Chem. 1997, 430, 25.

(18) Oldham, K. B.; Cardwell, T. J.; Santos, J. H.; Bond, A. M. J. Electroanal. Chem. 1997, 430, 39.

(19) Bento, M. F.; Thouin, L.; Amatore, C. J. Electroanal. Chem. 1998, 446, 91.

(20) Privat, C.; Trevin, S.; Bedioui, F.; Devynck, J. J. Electroanal. Chem. 1997, 436, 261.

(21) Evans, D. H.; Geraldo, M. D.; Montenegro, M. I.; Pletcher, D.; Slevin, L. J. Electroanal. Chem. 1997, 439, 115. 